\title{
Smoothing cubic spline approximation of silicon diode temperature sensors thermometric characteristics
}

\author{
O.M. Ivashchenko, Yu.M. Shwarts, M.M. Shwarts, D.P. Kopko, N.I. Sypko \\ V. Lashkaryov Institute of Semiconductor Physics, NAS of Ukraine, \\ 41, prospect Nauky,03028 Kyiv, Ukraine, phone/fax: +38(044)525-7463, \\ E-mail: iva@isp.kiev.ua; shwarts@isp.kiev.ua; mshwarts@isp.kiev.ua; kdp2007@ukr.net
}

\begin{abstract}
Shown in this paper is the efficiency of a smoothing cubic spline approximation for temperature response curves (TRC) of wide range silicon diode thermometers (SDTs). The offered calculation algorithm allows to describe TRC with a high accuracy and receive the interpolation tables with any temperature step. It allows essentially to expand SDT field of application.
\end{abstract}

Keywords: smoothing spline, spline approximation, thermodiode.

Manuscript received 29.06.10; accepted for publication 02.12.10; published online30.12.10.

\section{Introduction}

A great deal of attention is paid to silicon diode thermometers (SDT) in the modern world market of cryogenic semiconductor thermometers [1-3]. The main advantages of these thermometers are high adaptability to manufacture, stability, reproducibility and temperature sensitivity.

But the distinctive feature of SDT made by the various firms is nonlinearity of their temperature response characteristic (temperature sensitivity changes from unities up to tens or hundreds of $\mathrm{mV} / \mathrm{K}$ ) [1], which essentially complicates an approximation procedure. As a rule, the approximation of experimental TRC is carried out by the power polynomials determined by the least squares method (see, for example [4]). However, because of the computed error increase at a high power of the approximating polynomial, there is a necessity to split the sensor operation range by several subintervals with a subsequent labour-consuming "sewing together" procedure for the functions used in the approximation procedure. In [5], we have offered the method based on a combination of spline-interpolation and approximation by using the orthogonal Chebyshev polynomials. The method allows to avoid the procedure of splitting and to describe TRC with a uniform function keeping the necessary level of accuracy. The method provides the high accuracy of approximation $\pm 10 \mathrm{mK}$ with the polynomial of 200-th power in the 4.2 to $373 \mathrm{~K}$ and 25 th in the 30 to $373 \mathrm{~K}$ temperature ranges. But the application experience of the precision temperature measurement systems based on SDT requires to search for the approximation methods involving simpler functions. It would allow simplifying and reducing the cost of the sensor secondary equipment.

In recent years, many authors effectively applied the method of approximation based on the smoothing splines with free knots for description of the complex experimental curves $[6,7]$. Because of using the low power polynomials, the method provides a high accuracy of calculation. Furthermore, a smoothing character of the functions allows reducing the experimental error influence.

The purpose of this article lies in approbation of the free-knot smoothing cubic splines for approximation of TRC inherent to silicon diode thermometers.

\section{Samples and experimental technique}

The experimental batch of one-type SDT developed in V. Lashkaryov Institute of Semiconductor Physics was investigated. To reveal all TRC features and provide a high approximation accuracy, detailed calibration of sensors has been realized using the automated metrological system including gas-flow cryostat. 169 points were measured within the temperature range 4.2 to $373 \mathrm{~K}$ at the excitation current $1 \mu \mathrm{A}$, and 106 points - within the temperature range 30 to $373 \mathrm{~K}$ for current values 10 and $100 \mu \mathrm{A}$. The calibration technique is described in [8]. The temperature setting of the sensors was carried out in liquid helium and nitrogen vapors with the error of the temperature maintenance 
$\pm 0.01 \mathrm{~K}$. An error of the excitation current was $\pm 0.05 \%$, and the total error of the experimental data measurement in the temperature equivalent $\left(\Delta_{\exp }\right)$ was no more than $\pm 40 \mathrm{mK}$. The root-mean-square (RMS) value $\sigma_{\exp }$ of a random component in $\Delta_{\text {exp }}$ is less than $10 \mathrm{mK}$.

\section{Calculation of the temperature response curve}

To approximate the experimental data by the smoothing spline with a desired accuracy, one has to place $n$ knots $\tau_{j},(j=1, \ldots, n)$ on the temperature axis into the interval $\left[T_{1}, T_{\mathrm{N}}\right]$, where $T_{1}$ and $T_{\mathrm{N}}$ are the initial and final temperature calibration points, accordingly (Fig. 1). The positions of the former $\tau_{1}$ and latter $\tau_{n}$ knots coincide with the initial $T_{1}$ and final $T_{\mathrm{N}}$ temperature points of calibration. The task to search internal knots $\tau_{j}$, $j=2, \ldots, n-1$ (their number and place on the temperature axis) has no common solution and requires to take into account the kind of experimental data describing the function in each specific case.

For TRC approximation in the temperature interval $\left[T_{1}, T_{\mathrm{N}}\right]$, we have chosen the smoothing cubic spline that can be written as

$F(T)=\sum_{j=1}^{n+2} \alpha_{j} B_{j}(T)$,

where $\alpha_{j}$ are the spline coefficients; $B_{j}(T)-$ the $3-\mathrm{d}$ power B-spline calculated for $j$-th piece between knots.

This calculation of $F(T)$ is performed with account of the requirement of the sum-of-squares minimization for the sensor experimental voltage $U_{i}$ deviations from the spline $F(T)$ values in the temperature points $T_{i}$ :

$\min _{\tau, \alpha}\left(\sum_{i=1}^{N}\left(U_{i}-F\left(T_{i}\right)\right)^{2}\right)$.

The approximation error in the $i$-th temperature point is defined from the following expression:

$\Delta_{i}=\frac{U_{i}\left(T_{i}\right)-F\left(T_{i}\right)}{S\left(T_{i}\right)}$,

where $S\left(T_{i}\right)=d F\left(T_{i}\right) / d T$ are the calculated values of the sensor temperature sensitivity.

Taking into account presence of an essential random component in the data error, we use the following statistical parameters to estimate the approximation accuracy:

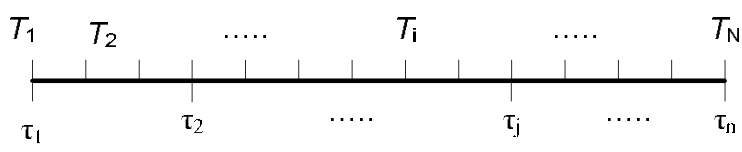

Fig. 1. Positions of experimental points $T_{i}$ and chosen spline knots $\tau_{j}$ on the temperature axis $\left[T_{1}, T_{\mathrm{N}}\right]$.
- the maximum approximation error

$M=\max _{i=1, \ldots, N}\left(\left|\Delta_{i}\right|\right)$

- the average approximation error

$\bar{\Delta}=\frac{\sum_{i=1}^{N} \Delta_{i}}{N} ;$

- the RMS approximation error

$\sigma=\sqrt{\frac{\sum_{i=1}^{N} \Delta_{i}{ }^{2}}{N-1} .}$

We suppose that the spline $F(T)$ will be acceptable, if the following criteria are met:

$M<\Delta_{\text {exp }} ;|\bar{\Delta}|<10 \cdot \Delta_{\text {exp }} ; 0.5 \cdot \sigma_{\exp }<\sigma<\sigma_{\exp }$.

The algorithm to calculate the function $F(T)$ is presented in Appendix.

\section{Results and discussion}

The experimental data of dependences $U(T)$, TRCs and temperature sensitivities that are calculated according to the offered algorithm for one of the investigated SDT GPI№20 are depicted in Fig. 2.
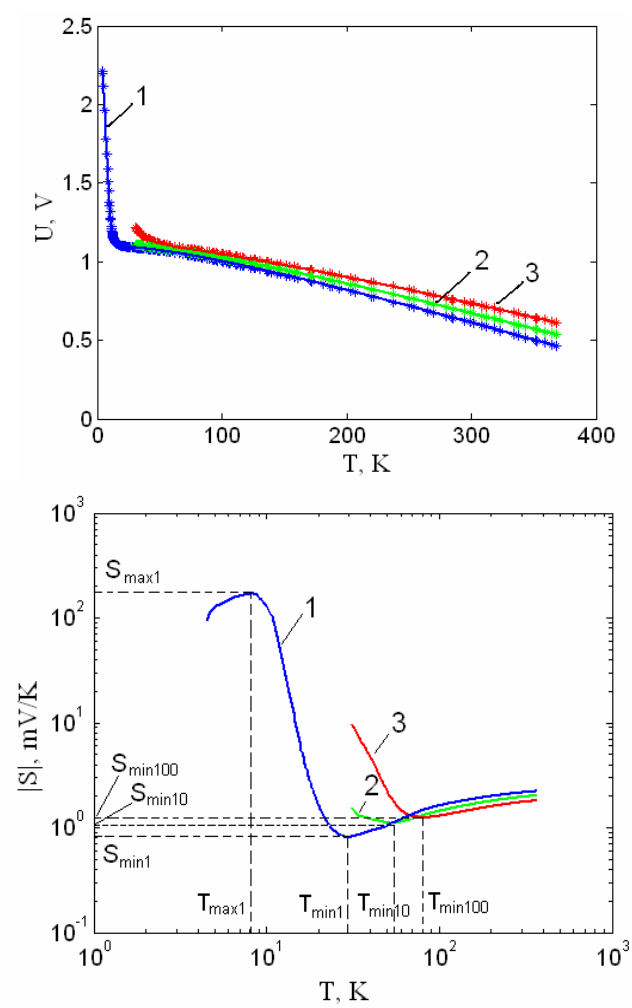

Fig. 2. The temperature response curve (a) and the temperature sensitivity (b) of the silicon diode thermometer GPI№20 (asterisks - experiment, lines - calculation). The excitation current, $\mu \mathrm{A}: 1-1 ; 2-10 ; 3-100$. 
TRC has a quasi-linear character within the range 100 to $373 \mathrm{~K}$ (Fig. 2a), and the sensor temperature sensitivities poorly depend on temperature (Fig. 2b). Nonlinearity is observed at $T<100 \mathrm{~K}$, and the sensitivities reach their minima at the temperatures $T_{\min 10}=54.25 \mathrm{~K} \quad\left(S_{\min 10}=-1.15 \mathrm{mV} / \mathrm{K}\right) \quad$ and $T_{\min 100}=84.63 \mathrm{~K} \quad\left(S_{\min 100}=-1.28 \mathrm{mV} / \mathrm{K}\right) \quad$ for the excitation current values $10 \mu \mathrm{A}$ (Fig. 2, curve 2) and $100 \mu \mathrm{A}$ (Fig. 2, curve 3), accordingly.

Besides, TRC for the excitation current $1 \mu \mathrm{A}$ has a more complicated form (Fig. 2, curve 1): the sensitivity minimum $S_{\min 1}=-0.82 \mathrm{mV} / \mathrm{K}$ and maximum $S_{\max 1}=-$ $173.5 \mathrm{mV} / \mathrm{K}$ are observed at the temperature points $T_{\min 1}=31.72 \mathrm{~K}$ and $T_{\max 1}=7.65 \mathrm{~K}$, accordingly.

The errors of the smoothing spline approximation in the operation temperature range for SDT GPI№20 are shown in Fig. 3.

Fig. 3 shows that TRC calculation for the current $1 \mu \mathrm{A}$ in the range 4.2 to $373 \mathrm{~K}$ with the maximum error $M=30 \mathrm{mK}$ requires $n=37$ knots that are arranged nonuniformly on the temperature axis. So, the number of the densely arranged knots equals to 23 in the range 4.2 to $100 \mathrm{~K}$ where TRC is characterized by the nonlinearity and the extrema in the temperature sensitivity are observed (Fig. 2, curve 1); and 14 knots are placed in a regular temperature intervals in the range of higher temperatures (100-373 K).
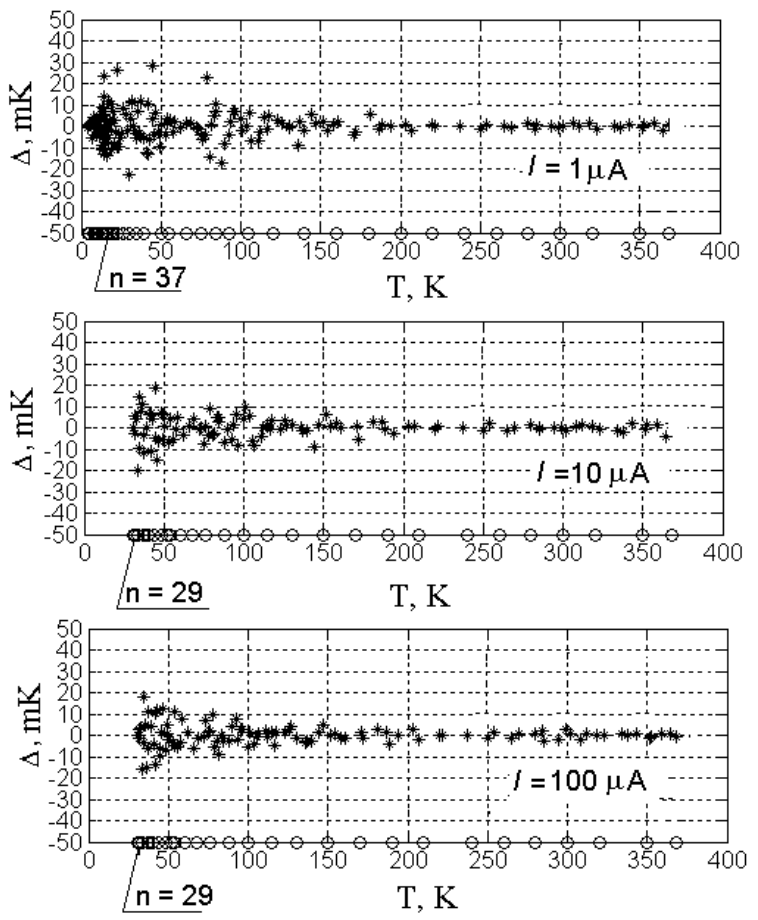

Fig. 3. The errors of the silicon diode thermometer GPI№20 experimental response curve approximation for the current values 1,10 and $100 \mu \mathrm{A}$ in the respective temperature range of calibration. The circles designate arrangements of the spline knots.
Table. The calculation results of the approximation statistical parameters for three samples of silicon diode thermometers.

\begin{tabular}{|c|c|c|c|c|}
\hline $\begin{array}{c}\text { SDT } \\
\text { number }\end{array}$ & $\begin{array}{c}\text { Current, } \\
\mu \mathrm{A}\end{array}$ & $M, \mathrm{mK}$ & $\bar{\Delta}, \mu \mathrm{K}$ & $\sigma, \mathrm{mK}$ \\
\hline \multirow{3}{*}{ GPI№20 } & 1 & 28 & -15 & 6.9 \\
\cline { 2 - 5 } & 10 & 20 & -1.5 & 5.5 \\
\cline { 2 - 5 } & 100 & 18 & -0.8 & 5.1 \\
\hline \multirow{3}{*}{ GPI№227 } & 1 & 35 & -14 & 9.0 \\
\cline { 2 - 5 } & 10 & 22 & -3.5 & 7.2 \\
\cline { 2 - 5 } & 100 & 18 & -1.2 & 5.1 \\
\hline \multirow{3}{*}{ GPI№220 } & 1 & 33 & -39 & 6.8 \\
\cline { 2 - 5 } & 10 & 19 & -4 & 5.5 \\
\cline { 2 - 5 } & 100 & 17 & -1.1 & 5.5 \\
\hline
\end{tabular}

To approximate the smoother curves at the currents 10 and $100 \mu \mathrm{A}$ in the range 30 to $373 \mathrm{~K}$ with the maximum error of $M=20 \mathrm{mK}$, we used $n=29$ knots also nonuniformly arranged along the temperature axis. For the attainment of an exhaustive description of the temperature sensitivity features (curves 2 and 3 in Fig. 2) the density of knots is higher in the 30 to $70 \mathrm{~K}$ temperature range.

TRCs of the batch consisting of 8 sensors were researched using the developed approximation algorithm. The calculation results of statistical parameters for the approximation of TRCs are summarized in Table for three samples.

Table shows that the highest maximum approximation error for the investigated SDT is observed at the excitation current $1 \mu \mathrm{A}$, and its magnitude does not exceed $35 \mathrm{mK}$. The calculated data completely satisfy the specified criterion (7). Thus, the obtained function $F(T)$ is acceptable for presentation of the experimental TRC.

\section{Conclusions}

An algorithm of approximation of TRCs inherent to SDT, which is based on free-knot cubic smoothing splines, is offered. The efficiency of the method is defined by fulfilling the offered criteria (7) that consider an experimental data error. The algorithm allows to present the temperature response curve with a high accuracy and to submit calculation data in analytical, table or graphic forms with any temperature steps, which essentially expands the field of application of diode thermometers.

\section{Appendix}

To arrange the initial set of knots, one should perform an analysis of the experimental dependence $U(T)$ and determine the temperature intervals (TI), on each of which TRC can be approximated by one spline piece. In what follows, one calculates the spline coefficients $\alpha_{\tau}=\left\{\alpha_{j}\right\}, j=1, \ldots, n+2$ using the following matrix expression: 


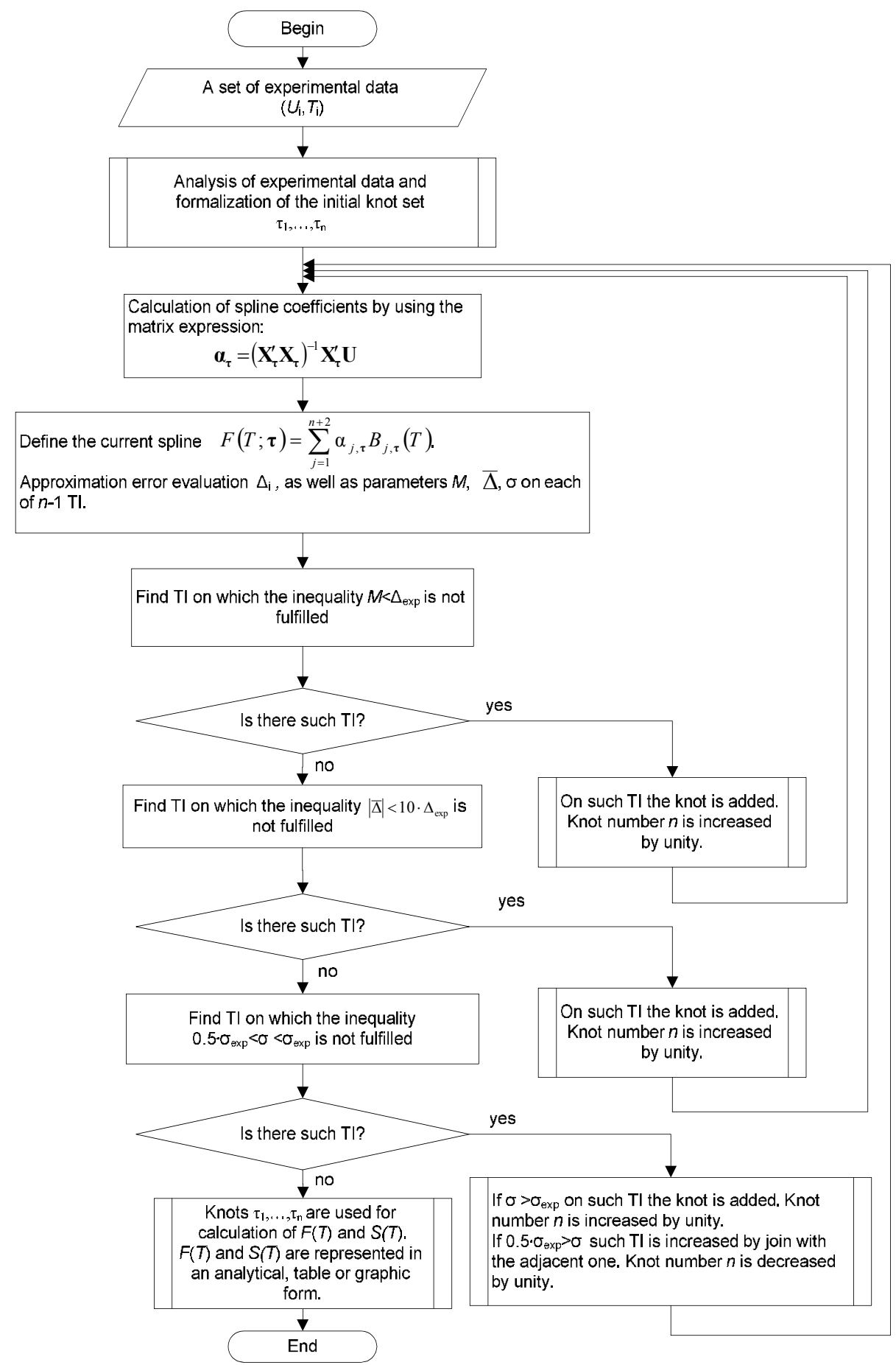

Fig. A1. The algorithm of temperature response curve and temperature sensitivity calculation by using the smoothing free-knot cubic spline.

$$
\mathrm{a}_{\tau}=\left(\mathbf{X}_{\tau}^{\prime} \mathbf{X}_{\tau}\right)^{-1} \mathbf{X}_{\tau}^{\prime} \mathbf{U},
$$

where $\mathbf{X}_{\tau}=\left\{\begin{array}{ccc}B_{1, \tau}\left(T_{1}\right) & \ldots & B_{n+2, \tau}\left(T_{1}\right) \\ \ldots & \ldots & \ldots \\ B_{1, \tau}\left(T_{N}\right) & \ldots & B_{n+2, \tau}\left(T_{N}\right)\end{array}\right\}$ is the

corresponding to the spline model matrix;

$\mathbf{U}=\left\{U_{i}\right\}, i=1, \ldots, N-$ the vector of measured voltages.
For the calculated coefficients (A1), the spline (1) is formed, and using the formulas (3)-(6) calculations of the errors $\Delta_{i}$ as well as parameters $M, \bar{\Delta}, \sigma$ are realized in each of $(n-1)$ TI.

In Fig. A1, the realized in MATLAB algorithm to calculate the TRC and temperature sensitivity of the SDT is presented. The algorithm is based on the cubic 
free-knot smoothing spline with specially chosen internal knots.

\section{References}

1. L.G. Rubin, Cryogenic thermometry: a review of progress since 1982 // Cryogenics, 37(7), p. 341356 (1997).

2. P.R.N. Childs, J.R. Greenwood, C.A. Long, Review on temperature measurement // Rev. Sci. Instrum., 71(8), p. 2959 (2000).

3. S.S. Courts, P.R. Swinehart, C.J. Yeager, A new cryogenic diode thermometer // Adv. in Cryogenic Eng. B, 47, p. 1620-1627 (2002).

4. Temperature Measurement and Control Catalog. Cryogenic Sensors, Instruments, and Accessories.
Published by Lake Shore Cryotronics, Inc., Westerville, OH (USA), 2004.

5. O.M. Ivashchenko, Yu.M. Shwarts, Approximation of thermometric characteristics inherent to silicon temperature diode sensors // Optoelektronika $i$ poluprovodnikovaya tekhnika, 38, p. 61-70 (2003), in Russian.

6. P. Ciarlini, D. Ichim, Free-knot cubic spline modelling in cryogenic thermometer calibration // Measurement, 39(9), p. 815-820 (2006).

7. N.I. Terikhova, Cubic smoothing splines // Matematicheskoye modelirovaniye, 2(8), p. 112118 (1990) in Russian.

8. Yu.M. Shwarts, O.M. Ivashchenko, M.M. Shwarts et al., Metrological support for diode thermometry // Pribory, 86(8), p. 5-11 (2007) in Russian. 